
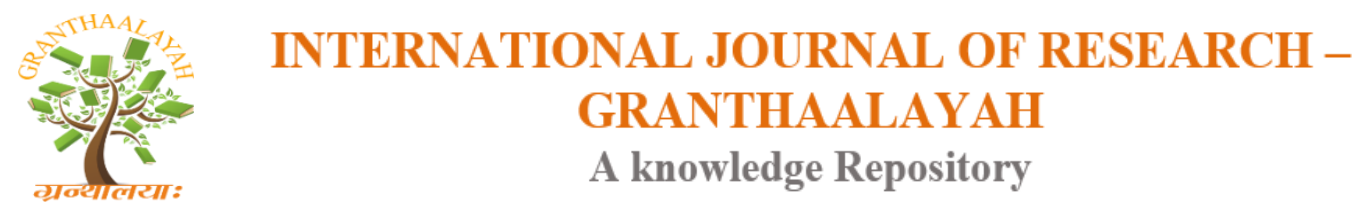

Science

\title{
ALLERGIC BRONCHOPULMONARY ASPERGILLOSIS (ABPA) - A CASE REPORT
}

\author{
Dr Binish Gulzar ${ }^{* 1}$ \\ ${ }^{* 1}$ Dr Chitralekha Saikumar Department of Microbiology, SBMCH, Chrompet. Chennai, India
}

\begin{abstract}
Case presentation: We present the case of a diabetic 73 year old male who worked as a farmer, with infiltrates in the right lower and middle lung lobes and with previous diagnosis of chronic obstructive bronchitis. Evaluation of our patient led to the diagnosis of allergic bronchopulmonary aspergillosis in the right lower and middle lobe. Fungal stains were positive for aspergillus. Based on the clinical, radiological and cytological findings, the patient was diagnosed with pulmonary aspergillosis and treated successfully with oral itraconazole and methylprednisolone. Systemic antifungal therapy plays an important role in preventing the morbidity and mortality. He was treated with oral methylprednisolone and itraconazole. Conclusion: Aspergillus infection after the inhalation of spores in the form of a hypersensitivity reaction and saprophytic colonization can be coexistent.
\end{abstract}

Keywords: Pulmonary aspergillosis; ABPA; Bronchitis; Antifungal.

Cite This Article: Dr Binish Gulzar. (2018). "ALLERGIC BRONCHOPULMONARY ASPERGILLOSIS (ABPA) - A CASE REPORT." International Journal of Research - Granthaalayah, 6(3), 27-29. https://doi.org/10.29121/granthaalayah.v6.i3.2018.1494.

\section{Introduction}

Aspergillosis refers to the spectrum of disease caused by Aspergillus species acquired by inhalation of airborne spores. This condition presents with a spectrum of illnesses, ranging from allergic reactions to colonization of pulmonary cavities to invasion and pyaemic spread to the brain, skin and other organs in the body $(1,2,3)$.There are different types of aspergillosis. Some types are mild, but some of them are very serious Pulmonary aspergillosis is classified as Invasive pulmonary aspergillosis (IPA), chronic necrotizing pulmonary aspergillosis (CNPA), Aspergilloma and Allergic bronchopulmonary aspergillosis (ABPA) (4).

Allergic bronchopulmonary aspergillosis (ABPA) causes inflammation in the lungs and allergy symptoms such as coughing and wheezing, but doesn't cause an infection. Allergic bronchopulmonary aspergillosis (ABPA) is a complex hypersensitivity reaction in patients with asthma, which occurs when bronchi are colonized by the Aspergillus species and most commonly due to Aspergillus fumigatus. The disease is characterized by type I and type III 
hypersensitivity reactions. The incidence may be as high as $6 \%$ of patients with asthma (5). Repeated episodes of bronchial obstruction, inflammation and mucoid impaction can lead to bronchiectasis, fibrosis and respiratory compromise.

\section{Case Presentation}

A 73 year old male, who worked as a farmer and was a non-smoker was admitted to the emergency department. He was a known case of COPD and diabetes. On admission to our hospital, he was afebrile. No lymphadenopathy was found. At the auscultation, prolonged expiration and weaker lung sounds over the right middle and basal field were heard. A chest radiograph showed the infiltrates with cavitation in the right middle lobe and in the lower lobe with consolidation of the latter. Laboratory investigations showed an elevated erythrocyte sedimentation rate of $84 \mathrm{~mm} / \mathrm{h}$. WBC count was normal. Pulmonary function tests showed reduced volumes. Sputum microscopy on $\mathrm{KOH} 10 \%$ mount reveals characteristic narrow septate hyaline hyphae with acute angle branching. Specimen was inoculated on SDA and incubated at $25^{\mathrm{O}} \mathrm{C}$. Macroscopic and LPCB mount of the specimen confirms Aspergillus species [fig1] . Histological examination of the bronchial biopsy specimen showed chronic inflammatory changes in bronchial mucosa with mononuclear cells and focally granulocytes infiltration. Histopathology showed non-specific inflammatory changes of bronchial mucosa. Cutaneous testing for the A. fumigatus antigen was strongly positive. Chest CT also showed tram-line shadows of bronchial wall thickening and cylindrical bronchiectasis in the middle lobe.

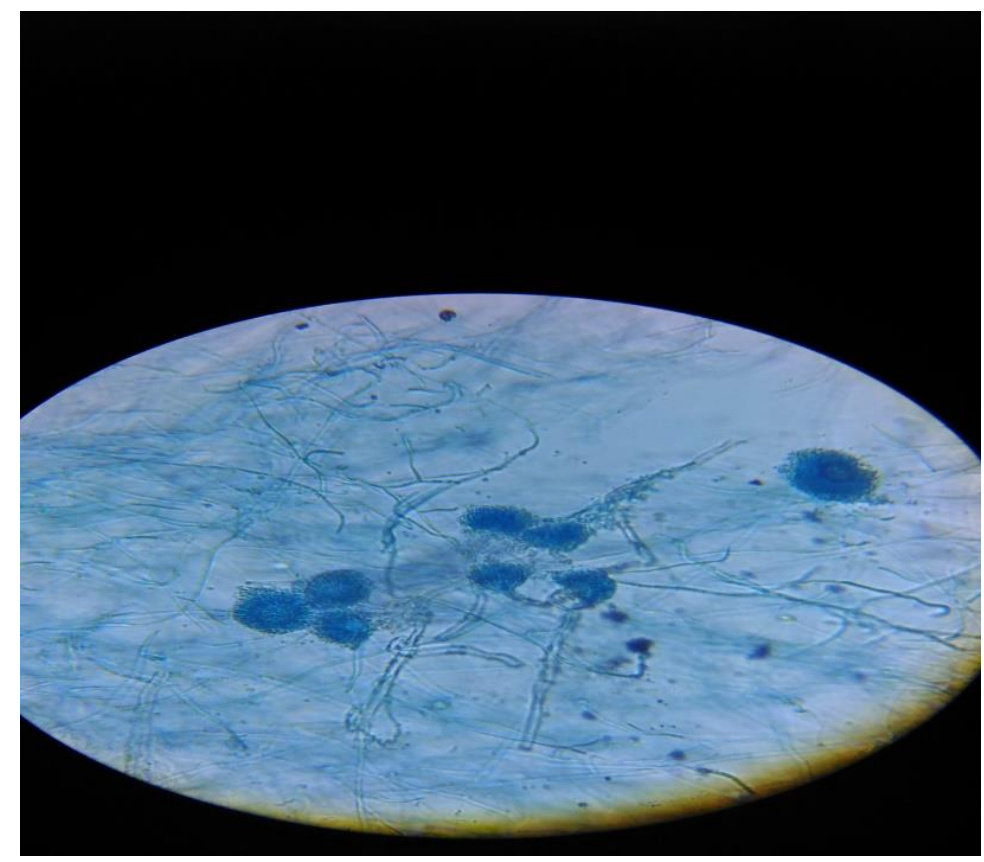

Figure 1: LPBC mount showing Aspergillus species

Since the patient was a farmer and therefore his exposure to an environment rich in the Aspergillus spores was high. Specific host susceptibility seems to be more important in the pathogenesis of ABPA than environmental factors. However, once the patient is sensitized a minor increase in spore concentration can cause symptomatic disease. Oral corticosteroids are the therapy of choice in ABPA. Itraconazole is the antifungal agent used for the therapy. 


\section{Conclusion}

We conclude that ABPA should be considered in a patient with repeated episodes of bronchial obstruction, inflammation and mucoid impaction along with complex hypersensitivity reaction that lead to respiratory compromise. And after corticosteroid and itraconazole treatment both subjective and objective improvements were experienced. Hence early treatment with systemic antifungal therapy should be initiated for preventing the morbidity and mortality.

\section{Refrences}

[1] Zmeili OS, Soubani AO. Pulmonary aspergillosis: a clinical update. QJM 2007; 100: 317-334.

[2] Thompson GR, Patterson TF. Pulmonary Aspergillosis. Semin Respir Crit Care Med. 2008; 28(2):103-110.

[3] Kousha M, Tadi R, Soubani AO. Pulmonary aspergillosis: a clinical review. (1) Eur Respir Rev 2011; 20: 121, 156-174.

[4] Soubani AO, Chandrasekar PH. The clinical spectrum of pulmonary aspergillosis. Chest 2002; 121: 1988-1999

[5] Leon EE, Craig TJ: Antifungals in the treatment of allergic bronchopulmonary aspergillosis. Ann Allergy Asthma Immunol 1999, 82:511-517

\footnotetext{
*Corresponding author.

E-mail address: drbinishh22@ yahoo.com
} 